\title{
Pavlovian-to-Instrumental Transfer in Alcohol Dependence: A Pilot Study
}

\author{
Maria Garbusow ${ }^{\mathrm{a}}$ Daniel J. Schad ${ }^{\mathrm{a}}$ Christian Sommer ${ }^{\mathrm{c}}$ Elisabeth Jünger ${ }^{\mathrm{c}}$ \\ Miriam Sebold ${ }^{a}$ Eva Friedel $^{\mathrm{a}}$ Jean Wendt ${ }^{\mathrm{a}}$ Norbert Kathmann ${ }^{\mathrm{b}}$ \\ Florian Schlagenhaufa, d Ulrich S. Zimmermann ${ }^{c}$ Andreas Heinz ${ }^{a}$ \\ Quentin J.M. Huys ${ }^{f, g}$ Michael A. Rapp ${ }^{e}$
}

${ }^{a}$ Department of Psychiatry and Psychotherapy, Charité Campus Mitte, Charité Universitätsmedizin Berlin, ${ }^{\mathrm{b}}$ Institute for Psychology, Humboldt-Universität zu Berlin, Berlin, ' Department of Psychiatry and Psychotherapy, Universitätsklinikum Carl Gustav Carus, Technische Universität Dresden, Dresden, dMax Planck Institute for Human Cognitive and Brain Sciences, Leipzig, and e Social and Preventive Medicine, Department of Health and Sports Sciences, University of Potsdam, Potsdam, Germany; ${ }^{\mathrm{f}}$ Translational Neuromodeling Unit, Institute of Biomedical Engineering, University of Zurich and ETH Zurich, and ${ }^{9}$ Department of Psychiatry, Psychotherapy and Psychosomatics, Hospital of Psychiatry, University of Zurich, Zurich, Switzerland

\section{Key Words}

Pavlovian-to-instrumental transfer - Alcohol dependence · Human

\begin{abstract}
Background: Pavlovian processes are thought to play an important role in the development, maintenance and relapse of alcohol dependence, possibly by influencing and usurping ongoing thought and behavior. The influence of pavlovian stimuli on ongoing behavior is paradigmatically measured by pavlovian-to-instrumental transfer (PIT) tasks. These involve multiple stages and are complex. Whether increased PIT is involved in human alcohol dependence is uncertain. We therefore aimed to establish and validate a modified PIT paradigm that would be robust, consistent and tolerated by healthy controls as well as by patients suffering from alcohol dependence, and to explore whether alcohol dependence is associated with enhanced PIT. Methods: Thirty-two recently detoxified alcohol-dependent patients
\end{abstract}

E-Mail karger@karger.com

www.karger.com/nps

\section{KARGER}

and 32 age- and gender-matched healthy controls performed a PIT task with instrumental go/no-go approach behaviors. The task involved both pavlovian stimuli associated with monetary rewards and losses, and images of drinks. $\boldsymbol{R} \boldsymbol{e}$ sults: Both patients and healthy controls showed a robust and temporally stable PIT effect. Strengths of PIT effects to drug-related and monetary conditioned stimuli were highly correlated. Patients more frequently showed a PIT effect, and the effect was stronger in response to aversively conditioned CSs (conditioned suppression), but there was no group difference in response to appetitive CSs. Conclusion: The implementation of PIT has favorably robust properties in chronic alcohol-dependent patients and in healthy controls. It shows internal consistency between monetary and drug-related cues. The findings support an association of alcohol dependence with an increased propensity towards PIT.

2014 S. Karger AG, Basel

\section{Q.J.M. Huys and M.A. Rapp contributed equally to this paper.}




\section{Introduction}

Between 75 and $85 \%$ of alcohol-dependent patients relapse after detoxification $[1,2]$, despite their stated desire to remain abstinent, and even in the face of severe consequences during relapse $[3,4]$. Contextual cues may be particularly important in this process [5-7].

Pavlovian conditioning describes the process by which neutral cues acquire value by predicting the occurrence of a rewarding or punishing event. Such cues often occur in drug-taking environments, and previous studies on both animals and humans have described their role in the development, maintenance and relapse of alcohol dependence [8-10].

The dopamine system, which is influenced by most (if not all) drugs of abuse, is known to be key to one type of pavlovian conditioning termed 'habitual' or 'model-free' learning [11-14]. Model-free learning depends on iteratively updating expectations through prediction errors (discrepancies between expected and experienced rewards). Phasic dopamine cell firing is known to report such reward prediction errors $[15,16]$ and to be causally involved in pavlovian conditioning [17]. Recently, work on signtracking highlighted the importance of individual variations in this type of learning for addiction, with sign trackers, who rely more on 'model-free' learning based on phasic dopamine teaching signals $[18,19]$ being at increased risk for dependence [20,21]. Hence, variability in how subjects learn about pavlovian conditioned stimuli is becoming a neurobiologically, clinically and theoretically coherent account for one risk factor for dependence.

One paradigm formalizing the influence of pavlovian conditioned stimuli (CSs) on ongoing instrumental behavior is pavlovian-to-instrumental transfer (PIT) [22]. In general PIT, positive pavlovian cues enhance instrumental responses while negative pavlovian cues inhibit instrumental behavior (conditioned suppression) independently of specific outcomes while in specific PIT pavlovian cues associated with a specific outcome influence ongoing behavior with the same outcome only $[23,24]$. Animal studies on general PIT have shown the involvement of dopamine and the nucleus accumbens [23, 25]; PIT effects are reduced under dopamine antagonists [26-28], and drug-related cues enhance both behavioral [29-32] and neuronal [33] PIT effects in drug-treated groups. In humans, PIT effects are also measurable and involve a similar set of neural structures (including the amygdala and nucleus accumbens) [34-40]. General PIT thus has important parallels with sign tracking.

However, how individual differences in PIT are associated with alcohol dependence in humans is not known.
Such paradigms involve multiple stages, and are thus both lengthy and complex. Their reliability has not been examined in patient populations. Thus, there is a need for tasks that measure the individual subject's tendency towards model-free pavlovian learning. We here present a PIT task (adapted from Huys et al. [36] and Geurts et al. [39]) that measures pavlovian influences on instrumental approach/ no approach behavior. The paradigm carefully matches the instrumental expectations of the actions themselves. It includes both pavlovian stimuli conditioned to predict monetary outcomes, and drug-associated versus neutral stimuli. We expected to see stronger PIT effects both for stimuli predicting monetary outcomes, and for alcohol-related pictures in patients suffering from alcohol use disorder (AUD).

\section{Methods}

\section{Participants}

In a bicenter study, we tested 32 recently detoxified alcohol-dependent patients $\left(M_{\text {age }}=42.13, S D_{\text {age }}=9.78 ; 29\right.$ males $)$ and 32 ageand gender-matched healthy controls $\left(M_{\text {age }}=42.34, S D_{\text {age }}=10.29\right.$, 29 males). Exclusion criteria were: major psychiatric or neurologic disorders, a history of any dependence syndrome or current substance abuse (assessed by drug urine testing) except nicotine dependence in healthy controls and nicotine and alcohol dependence in patients, intake of medications or drugs known to interact with the central nervous system within at least 4 half-lives after the last intake (including detoxification treatment with benzodiazepines or clomethiazole). We included right-handed subjects only. Alcohol-dependent patients had a minimum of 3 years of AUD, $72 \mathrm{~h}$ to 21 days of abstinence, as well as a low to moderate severity of withdrawal symptoms (revised Clinical Institute Withdrawal Assessment for Alcohol (CIWA-Ar) $<3$ [41]). To ascertain inclusion and exclusion criteria, we used a computer-based clinical interview (Composite International Diagnostic Instrument (CIDI) [42]). All participants gave their written informed consent to participate; ethical approval for the study was obtained from the ethics committee of the Charité Universitätmedizin Berlin and Universitätklinikum Dresden. Participants received a monetary compensation for study participation.

\section{Setting}

The task was programmed using Matlab with the Psychophysics Toolbox Version $3[43,44]$ extension. It was presented on a Dell laptop screen (instrumental training, forced choice) and on a projector via a mirror system (pavlovian training and PIT). Participants performed the PIT task in a functional magnetic resonance imaging (MRI) setting as part of a larger study examining neural correlates of learning mechanisms in alcohol dependence (see www.lead-studie. de). We here report behavioral data only. The instrumental training was conducted before the scanner session, the pavlovian and PIT part inside the MRI scanner, and the forced choice task after the scanning session. Participants wore MRI compatible Siemens headphones; the volume was individually adapted. Responses were made on a $1 \times 4$ Current Design MRI compatible response box button using the right index finger (instrumental response in training and transfer) or 2 buttons using the left and the right index fingers (forced choice). 


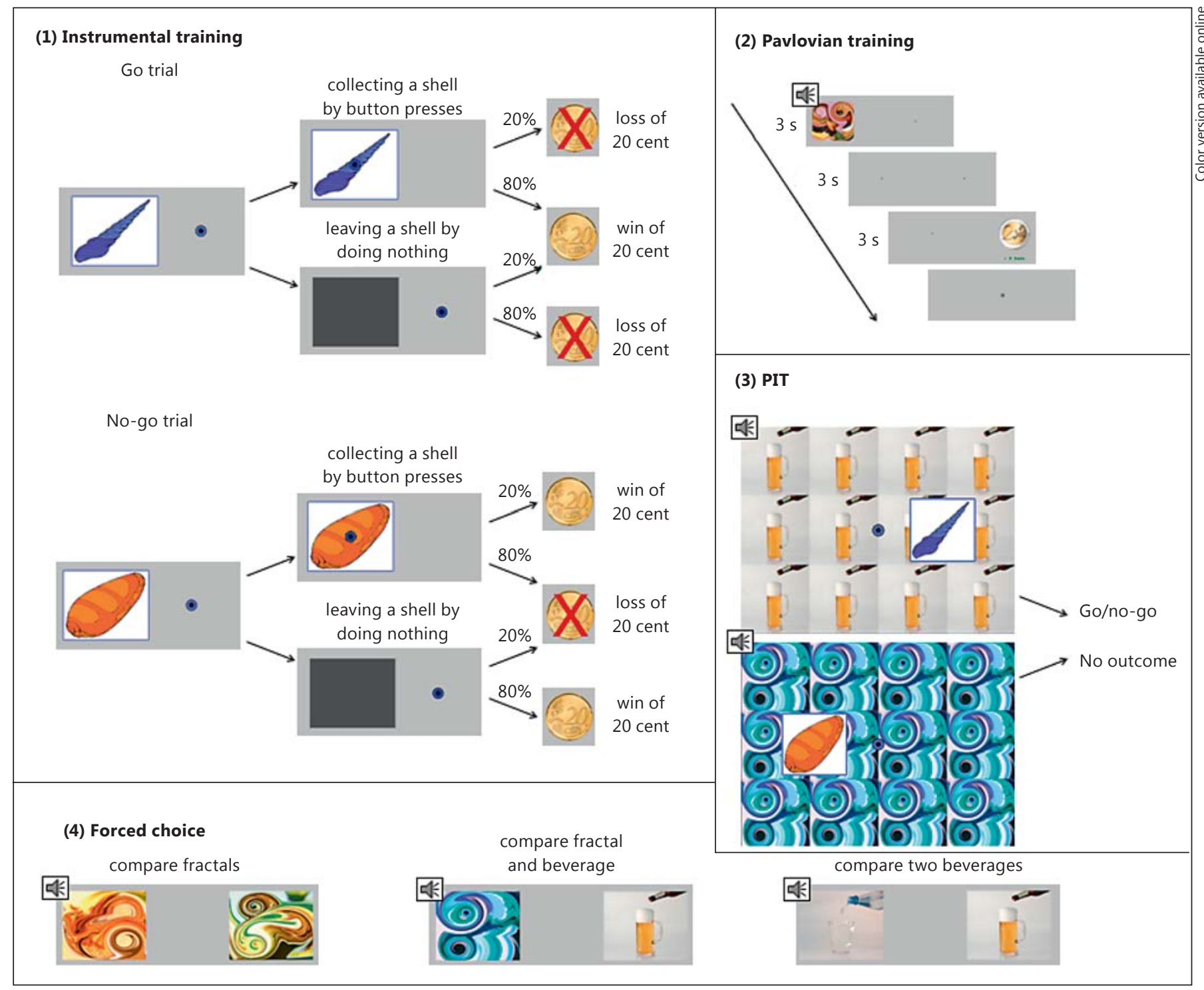

Fig. 1. The PIT paradigm consisted of 4 parts: (1) instrumental training - go or no-go responses, reinforced by probabilistic outcomes; (2) pavlovian training - audiovisual compound cues ('fractal CSs') were associated with 1 out of 5 outcomes; (3) PIT - subjects performed the instrumental task in nominal extinction, i.e.

\section{PIT Paradigm}

The PIT paradigm (adapted from Huys et al. [36] and Geurts et al. [39]) consisted of 4 parts: (1) instrumental training, (2) pavlovian training, (3) PIT and (4) a forced choice task (fig. 1).

\section{Instrumental Training}

During instrumental training, the subjects were instructed to collect shells by button presses. One instrumental stimulus appeared on the left or right side of the screen (counterbalanced). The subject's task was to move a dot toward the stimulus by repeated button presses in order to collect it or to do nothing within $2 \mathrm{~s}$. no explicit outcomes were presented; the background was tiled with either drink Cs (top) or fractal CSs (bottom); (4) at the end, subjects performed forced choices between 2 fractal CSs, a fractal and a drink CS or 2 drink CSs.
These two instrumental choices resulted in monetary wins or losses, presented immediately after each trial via a picture of a 20 -eurocent coin for $1.5 \mathrm{~s}$. Feedback was probabilistic. A 'good' shell was rewarded in $80 \%$ and punished in $20 \%$ of trials if collected (go) and vice versa if not collected. A 'bad' shell was rewarded in $80 \%$ and punished in $20 \%$ of the trials if not collected (no-go) and vice versa if collected. The classification of a shell as 'good' or 'bad' and the order of stimulus presentation were randomized over participants. All 6 shells consisted of 2 different colors, were highly discriminable with respect to color and shape, and had comparable visual features (such as size and resolution). 
The instrumental training comprised a minimum of 60 trials. To match for instrumental performance, we established a learning criterion ( $80 \%$ correct choices over 16 trials). Instrumental training ended once participants reached the learning criterion or at a maximum of 120 trials.

\section{Pavlovian Conditioning}

At the beginning of each trial, a compound CS consisting of a multicolored fractal-like distorted image on the right or left side of the screen (counterbalanced across subjects, fixed within subjects) paired with a pure tone via headphones was presented for $3 \mathrm{~s}$. Henceforth, the combined audiovisual CSs involving fractals will be referred to as 'fractal CSs'. After a delay of $3 \mathrm{~s}$ (presenting 2 fixation crosses at the 2 potential CS locations), a coin (unconditioned stimulus, US) was presented for a further $3 \mathrm{~s}$ on the opposite side. Subjects were instructed to observe the CSs and USs and to memorize the pairings. The CS-US association was deterministic and fixed within, but randomized across subjects. The set of stimulus pairings consisted of 2 positive CSs paired with +2 and +1 euros, 1 neutral CS paired with 0 euros (picture of a zero) and 2 negative CSs paired with -1 and -2 euros (coins with superimposed red cross). Subjects completed 80 trials.

To ensure that the pavlovian stimuli had comparable subjective ratings and visual features, we created a set of 20 pictures by distorting and recoloring food photographs with the GIMP software (http://www.gimp.org/). All pictures had equal mean luminance values, and equal root-mean-square contrasts of the luminance values [45]. An independent sample of 75 people (42 females, $M_{\text {age }}=29.8$ and $S D_{\text {age }}=12.2$ ) rated the pictures on a 7-point-Likert scale according to the 4 dimensions pleasure, arousal, beauty and alcohol craving. We chose 5 highly discriminable CSs with respect to color and shape out of those pictures with equal ratings on all subjective rating dimensions.

\section{Pavlovian-to-Instrumental Transfer}

The subjects performed the instrumental task again with either fractal or drink CSs tiling the background. No outcomes were presented, but the subjects were instructed that their choices still counted towards the final monetary outcome. There were 4 drink CSs: 2 were alcoholic (photographs of the participant's favorite alcoholic drink) and 2 neutral (photographs of a water glass; all with homogeneous white background). Drink stimuli were paired with the sound of pouring a drink into a glass. Participants completed 162 trials whereby 9 different background stimuli (5 CSs previously paired with money, 4 pictures of beverages) were shown 18 times each in a pseudorandom order. The response window was 3 $s$ with 2-6 s as interstimulus interval (individually exponentially distributed jitter).

\section{Forced Choice Task}

Finally, the participants completed a forced choice task. The subjects had to choose 1 of 2 compound CSs. All possible CS pairings were presented 3 times in an interleaved, randomized order, and stimuli were presented one at a time for $2 \mathrm{~s}$. Slow responses led to a reminder requesting faster responses. We used these data to verify the acquisition of pavlovian expectations.

\section{Data Analysis}

Data were analyzed using Matlab 2011a [46] and IBM SPSS Statistics 20 [47]. When performing ANOVAs, we report Green-
house-Geisser corrected statistics where appropriate (after testing for homogeneity and sphericity using Box's test of equality of covariance and Mauchly's test of sphericity).

Instrumental Performance. We computed a $2 \times 2$ repeated measures ANOVA for analyzing the number of button presses, both for the instrumental training and in the PIT task. There was one within-subjects factor for approach go versus approach no-go and one between-subjects factor group (healthy controls vs. patients).

Pavlovian Training. Individual acquisition of pavlovian associations was assessed by the fraction of correct answers on the forced choice task using a $\chi^{2}$ test. Group comparisons were performed with 2-sample t tests or signed rank tests, as appropriate.

PIT. PIT data for fractal CSs and drink CSs were assessed separately. The number of button presses was averaged for each instrumental-pavlovian stimulus combination. We used repeated measures ANOVAs with the number of button presses as dependent variable and 2 factors (CS and group). The group factor was between subject. The CSs were coded as within-subject factors. For fractal CSs there were 5 levels corresponding to each outcome, for drink CSs there were 2 levels corresponding to alcohol or nonalcohol. Individual PIT effects were quantified by regressing the mean number of button presses on the value of the CSs and retaining the slope. This was done across all CSs to estimate the overall PIT effect, across neutral and negative CSs for conditioned suppression and across neutral and positive stimuli for the positive PIT effect. Simple group comparisons were performed by Wilcoxon signed rank test if conditions for $t$ tests were violated.

To estimate the stability of the PIT effect, we computed the individual PIT effect slopes for the first and second halves of the experiment separately and then correlated these (Pearson linear correlation for gaussian and Spearman rank coefficient for nongaussian data). Finally, we conducted correlation analyses between the individual regression coefficients calculated for fractal and drink CSs for each subject.

\section{Results}

\section{Instrumental Behavior}

Half the patients achieved the instrumental training criterion (after 82.1 trials on average) and 20 out of 32 controls (after 74.7 trials on average). The remaining subjects performed all 120 trials of the instrumental training. Overall, the subjects learned to press more when approach was more rewarded (go condition) and to press less when not approaching was more rewarded (no-go condition; $\mathrm{F}_{1,62}=54.11, \mathrm{p}<0.001$; fig. $2 \mathrm{a}$ ). This difference was stably maintained during PIT $\left(\mathrm{F}_{1,62}=33.27, \mathrm{p}<\right.$ 0.001; fig. 2b). There was no effect of group either during instrumental training $\left(\mathrm{F}_{1,62}=2.42, \mathrm{p}=0.13\right)$ or during the PIT part of the experiment $\left(\mathrm{F}_{1,62}=2.26, \mathrm{p}=0.14\right)$, and there were no interactions between condition (go/no-go) and group in either the training or PIT part $\left(\mathrm{F}_{1,62}=0.84\right.$, $\mathrm{p}=0.37$, and $\mathrm{F}_{1,62}=0.01, \mathrm{p}=0.94$, respectively). 
Fig. 2. Instrumental performance. Mean number of button presses: subjects learned to collect a 'good' shell (reward when collected, go condition) and to leave a 'bad' shell (reward when not collected, no-go condition). Results are shown for the instrumental learning task (a) and the PIT task (b). In the PIT task, the subjects had to perform the instrumental response they learned in the instrumental training without direct feedback. Black bars represent standard errors of the mean. ${ }^{* * *} \mathrm{p}<0.001$. Fig. 3. Pavlovian conditioning. a Successful pavlovian learning is visible in the forced choice task indicating a high preference for the fractals associated with higher outcomes. Bars show the mean probability of a better stimulus for those subjects performing above chance (black dots). Crosses show subjects performing at or below chance. b Choice probabilities involving drink CSs in the forced choice task, comparing fractal CSs with drink CSs or comparing 2 drink CSs. Alc = Alcoholic drink; $\mathrm{Wtr}=$ water; Alc $>\mathrm{CS}=$ probability of choosing drink of fractal CS; Wtr $>$ CS = probability of choosing water over fractal $\mathrm{CS}$; Alc $>$ Wtr = probability of choosing alcoholic over water CS. Black bars represent standard errors of the mean. ${ }^{* *} \mathrm{p}<0.01$.
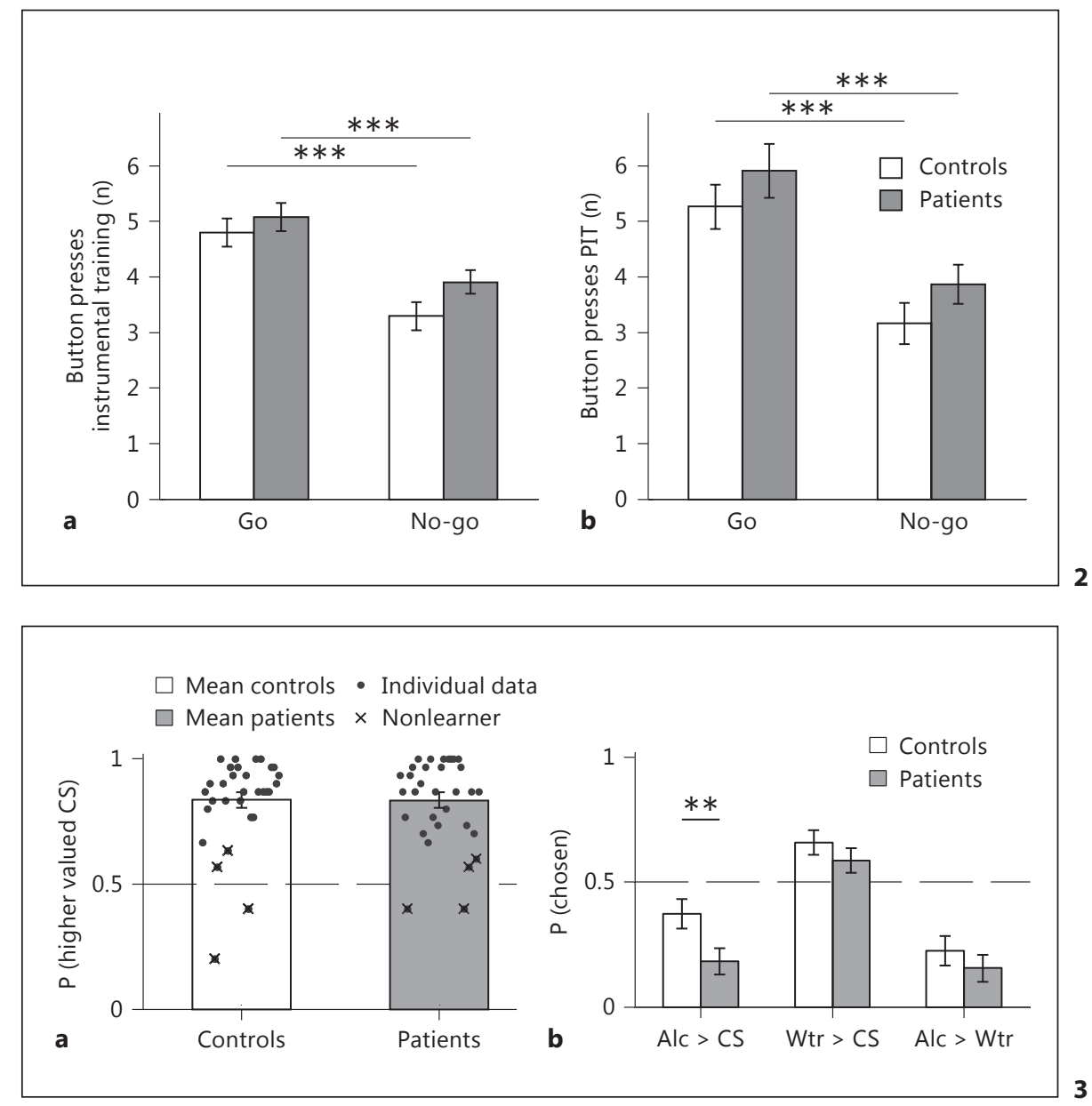

\section{Forced Choices of Fractal CSs}

To assess the acquisition of pavlovian values, we analyzed preferences in the forced choice trials involving only pairs of fractal CSs (fig. 3a). Twenty-eight out of 32 patients and 27 out of 31 controls (in 1 healthy control, the forced choice data were missing), preferred highervalued fractal CSs overall (with individual $\mathrm{p}<0.05$ ). $\mathrm{Pa}$ tients and controls chose the better of the two fractals in $88.21 \pm 0.02$ and $89.42 \pm 0.03 \%$ of the trials, respectively. There was no significant group difference $(\mathrm{p}=0.95$, $\mathrm{z}=-0.06$, rank sum $=987$, Wilcoxon rank sum test). The one healthy control with missing data in forced choices and the 8 subjects who failed to show preference for fractal CSs with higher associated value were excluded from the PIT analyses as pavlovian conditioning was uncertain.

\section{Forced Choices Involving Beverage CSs}

Subjects also performed forced choices involving pictures of their favorite drink and/or water and of drinks paired with fractal CSs (fig. 3b). Patients chose fractals over alcoholic CSs more than controls $(\mathrm{p}<0.01, \mathrm{z}=-2.65$, rank sum $=639$, Wilcoxon rank sum test; Bonferroni correctable for 3 comparisons) but otherwise did not differ from controls.

\section{PIT Effects: Fractal CSs}

There was a significant main effect of fractal CSs $\left(\mathrm{F}_{1.301,53}=28.14, \mathrm{p}<0.001\right.$, Greenhouse-Geisser corrected); but this did not differ between groups (fractal CS $\times$ group $\mathrm{F}_{1.301,53}=0.78, \mathrm{p}=0.412$, Greenhouse-Geisser corrected; fig. 4a). Exploratory analyses of the fractal CS effects on button press responses only during go or during no-go also failed to yield group differences. The effect of fractal CSs was present both in subjects who had $\left(\mathrm{F}_{1.267,53}=14.76, \mathrm{p}<0.001\right.$, Greenhouse-Geisser corrected) and who had not $\left(\mathrm{F}_{1.33,53}=11.87, \mathrm{p}<0.01\right.$, Greenhouse-Geisser corrected) reached the instrumental criterion. 


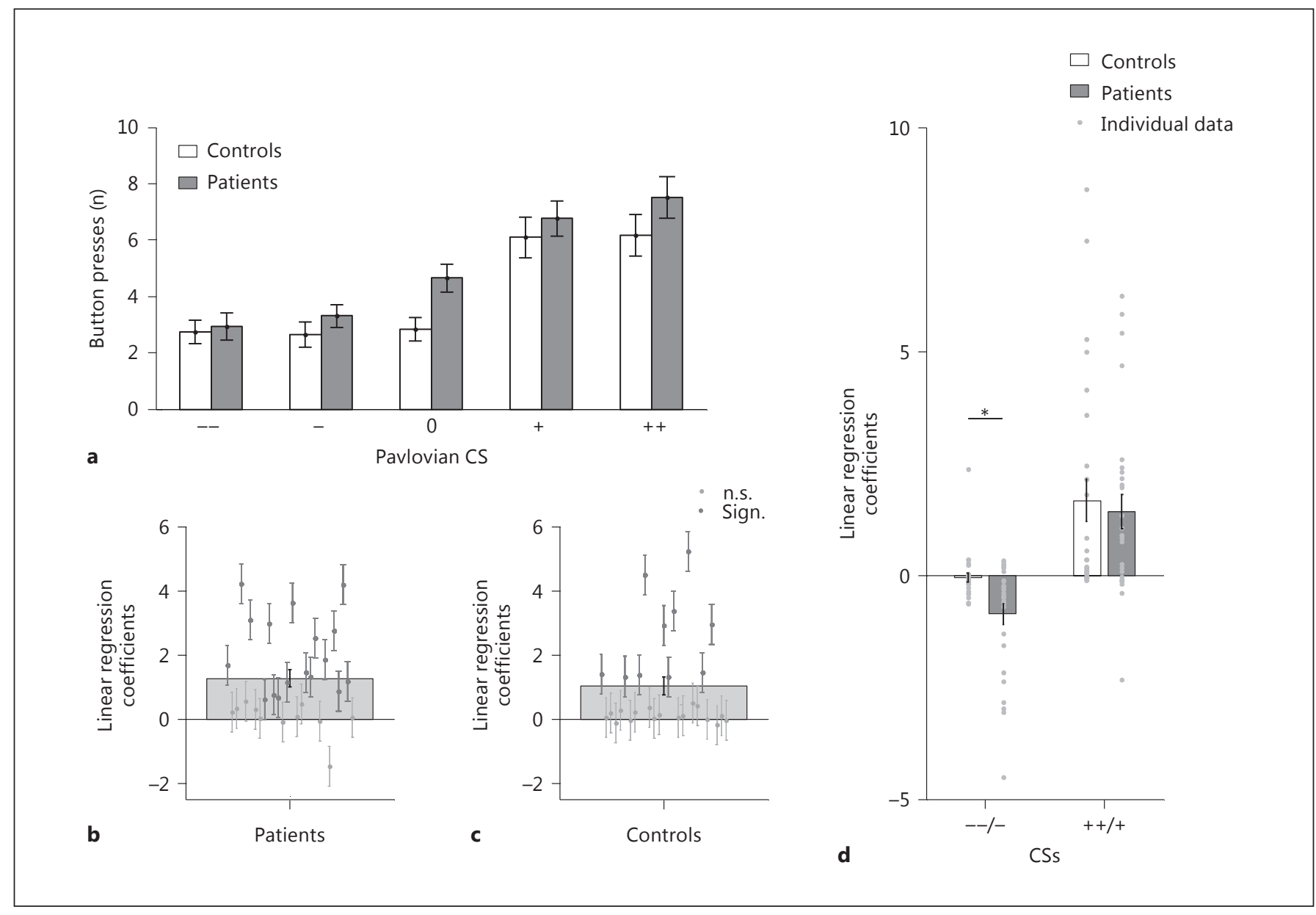

Fig. 4. a-d PIT effects for experimentally conditioned CSs. a The mean number of button presses increased with increasing value of the conditioned CS in the background. b, c Group mean (solid gray bar) and individual (dots) linear regression coefficient. Light gray dots represent individuals not showing a significant PIT effect, dark gray dots represent individuals with a significant effect ( $\mathrm{p}<$ 0.05). Error bars represent standard errors of the mean. b Patients. c Controls. d Comparing valenced (positive and negative CSs) to neutral CSs shows a group difference only on the aversive side. $* \mathrm{p}<0.05$.
As a measure of individual PIT effects, we calculated linear regression coefficients between the number of button presses and the fractal CS value for each individual separately. This was individually significant ( $\mathrm{p}$ values $<0.05)$ in 17 out of 28 patients $(61 \%$; fig. $4 \mathrm{~b})$ and in 10 out of 27 healthy controls (37\%; fig. 4c). This fraction was trendwise different between groups $\left(\chi^{2}=3.08\right.$, $\mathrm{p}=0.08$ ).

PIT is usually only considered with appetitive CSs, while the aversive side is typically examined in separate experimental setups in conditioned suppression. This motivates a separate analysis of the positive and negative limbs of the experiment. We therefore computed separate linear regression coefficients for the positive and the neg- ative CSs, always including the neutral CS. Patients showed a stronger effect of negatively valued fractal CSs (conditioned suppression) than controls ( $\mathrm{p}<0.05, \mathrm{z}=$ -2.34 , rank sum $=644$; Bonferroni correctable for 2 comparisons; fig. 4d). Groups did not differ in terms of the effect of positive fractal CSs $(\mathrm{p}=0.97, \mathrm{z}=-0.04$, rank sum $=781$ ).

\section{PIT Effects: Beverage CSs}

Two outliers (1 patient and 1 control) were outside the range of 3 standard deviations around the mean and therefore removed. There was a main effect of drink CSs, with alcohol CSs reducing responses compared to water CSs $\left(F_{1,51}=10.4, p<0.01\right.$; fig. 5a). The effect did not differ 


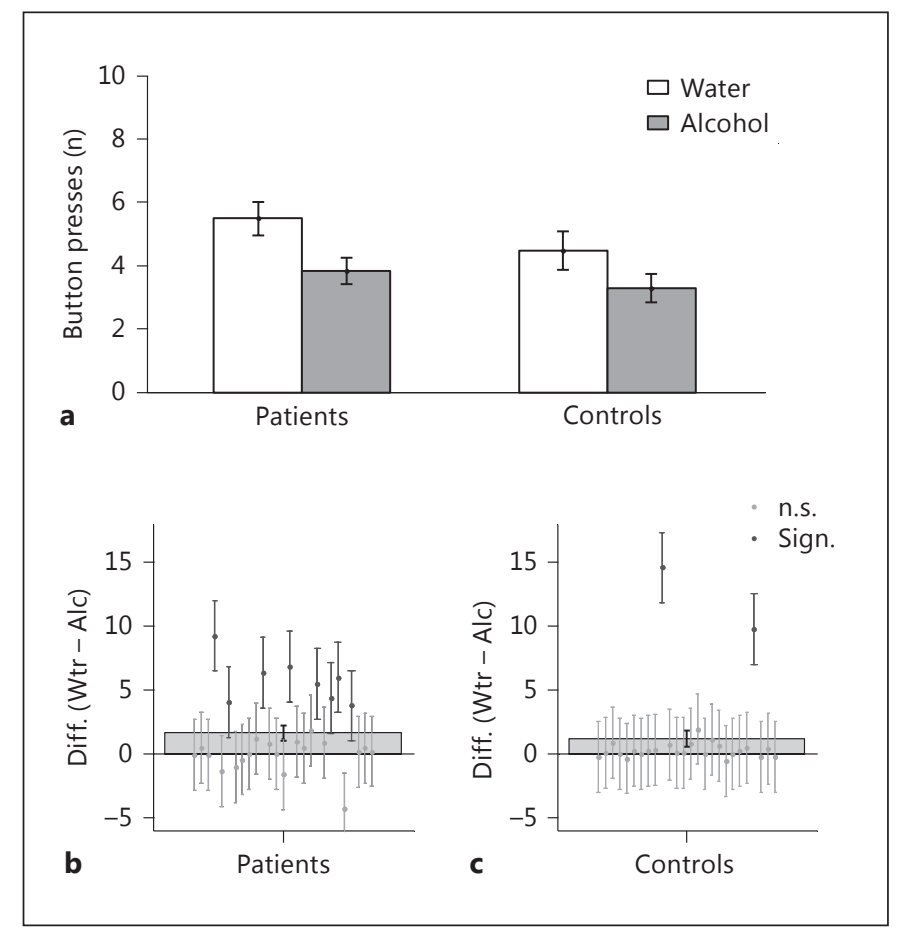

Fig. 5. a-c PIT effect for beverage CSs. a Alcoholic drink CSs reduce responding compared to neutral water drink CSs. b, c The mean (light gray bar) and individual (dots) difference (Diff.) between water (Wtr) and alcohol (Alc) cues. Light gray dots represent individuals (patients in $\mathbf{b}$, controls in $\mathbf{c}$ ) not showing a significant PIT effect, dark gray dots represent individuals with a significant effect $(\mathrm{p}<0.05)$. Error bars represent standard errors of the mean.

between groups (drink CS $\times$ group; $\mathrm{F}_{1,51}=0.28, \mathrm{p}=0.599$ ) and was present both in patients $\left(\mathrm{F}_{1,26}=7.79, \mathrm{p}<0.05\right)$ and marginally in controls $\left(\mathrm{F}_{1,25}=3.28, \mathrm{p}=0.082\right)$.

We again computed individual PIT scores by fitting linear regressions. These were individually significant in 8 of the 28 patients (28.57\%; fig. 5 b) and in 2 out of 27 healthy controls (7.41\%; fig. 5c). Mirroring the fractal CS result, there was a group difference in the proportion of subjects showing a drink CS effect $\left(\chi^{2}=4.14, \mathrm{p}=0.04\right)$.

\section{Temporal Stability of PIT Effects}

Individual regression coefficients for PIT effects with fractal CSs were computed separately on the first and second half of the experiment. The correlation between these was very high $(\rho=0.79, p<0.001$, Spearman; fig. $6 a)$, suggesting a temporally very stable PIT effect. This remained true when considering patients $(\rho=0.78, p<0.001$; Spearman) and controls ( $\rho=0.77, \mathrm{p}<0.001$; Spearman $)$ separately.

Pavlovian-to-Instrumental Transfer in Alcohol Dependence
Similarly, PIT effects for drink CSs were stable between first and second halves when collapsing across groups ( $\rho=0.34, p<0.05$, Spearman; fig. $6 b)$ or when considering patients ( $\rho=0.52, p<0.01$, Spearman) alone. In contrast, there was no evidence for a stable drink CS PIT effect in controls ( $\rho=0.14, p=0.5$, Spearman), though the group difference failed to reach significance (Fisher's $\mathrm{z}=1.52$, 2-tailed $\mathrm{p}=0.13$ ).

\section{PIT Effects for Fractal and Drink CSs Are Correlated}

Finally, we asked whether subjects who showed a PIT effect in response to fractal CSs would also tend to show a PIT effect in response to drink CSs. Individual PIT effects in response to drink and fractal CSs were highly correlated. This was true when collapsing across groups $(\rho=$ $0.45, \mathrm{p}<0.001$, Spearman; fig. 7 ) or when considering patients $(\rho=0.47, p<0.05$, Spearman) or controls ( $\rho=$ $0.40, \mathrm{p}<0.05$, Spearman) individually.

\section{Discussion}

The current study was a pilot study to establish the feasibility of measuring the influence of pavlovian processes on ongoing behavior in alcohol-dependent patients in comparison to healthy controls (see www.leadstudie.de). We chose PIT due to its substantial preclinical evidence base relating both to addiction and dopaminergic processes $[22,48]$. However, it is a complex paradigm, which has not always shown strong behavioral effects [35] (particularly inside the MRI scanner), and as such it was necessary to first establish the feasibility of such a task in a challenging patient population. The 3 main conclusions of this pilot study are that (i) PIT effects can indeed be measured behaviorally in a stable and reliable manner, (ii) that PIT effects in response to drug-relevant and monetary CSs are consistent and (iii) that recently detoxified patients suffering from AUD and healthy controls differ modestly in the propensity to show PIT effects and in the strength of conditioned suppression (negative PIT).

For task measures to have clinical validity, it is critical that they are reliable and valid within the whole cohort, and show substantial variability between individuals in a manner that is consistently related to the task construct. First, we found that individual PIT effect strength is stable over time, being highly correlated between the two halves of the experiment. Although this is very encouraging, it does not replace the need for assessing test-retest reliability with an intervening inter- 
Fig. 6. a, b Correlation between regression coefficients of individual PIT slopes for first and second halves of the PIT experiment concerning conditioned CSs (a) and beverage stimuli (b) in the background.

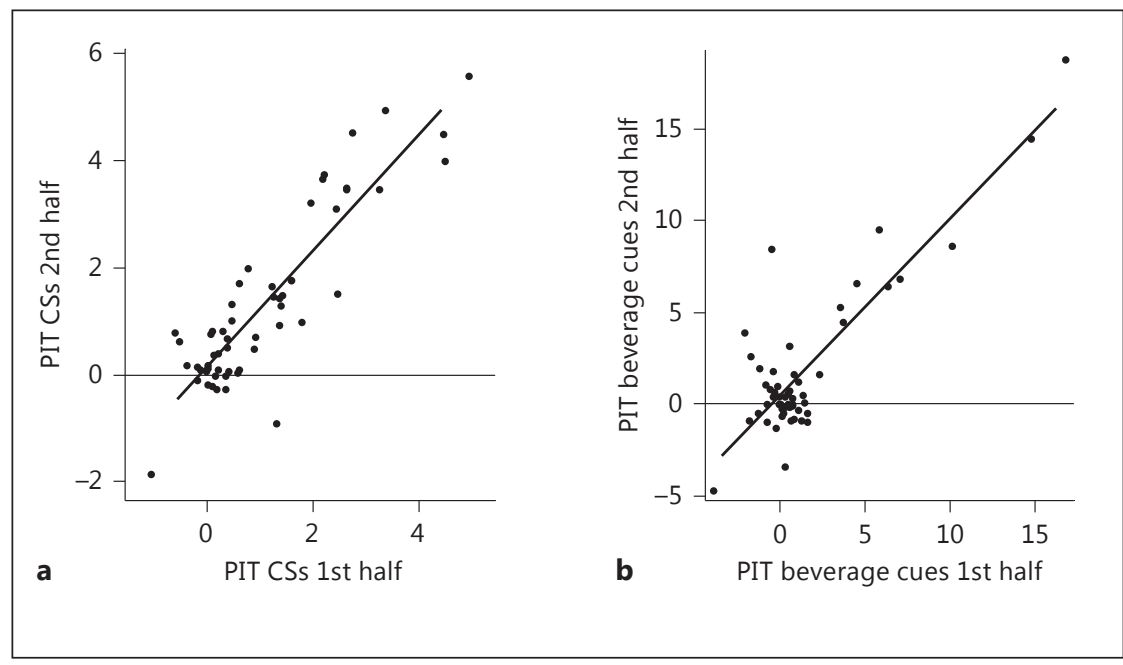

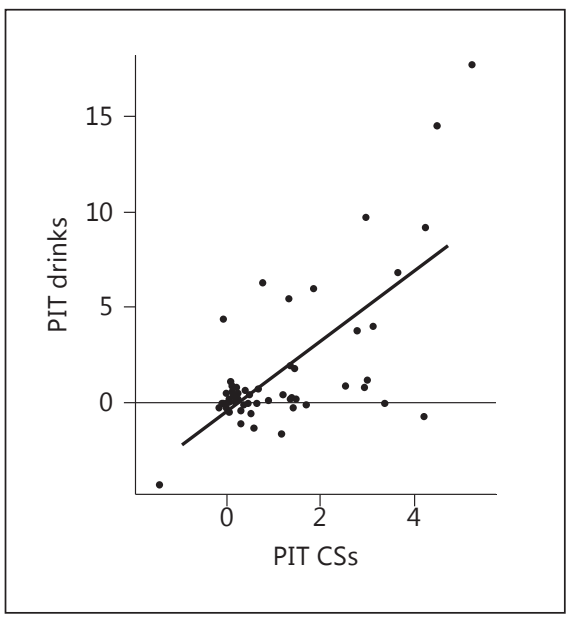

Fig. 7. Correlation between regression coefficients of individual PIT slopes for trained CSs and beverage stimuli in the background.

val. The fact that the drink CS effect in controls was not stable is most likely due to poor power as only 2 subjects showed an individually significant effect. Overall, stability was comparable in patients and controls. Second, we attempted to measure the internal consistency of the PIT effect by correlating the effects of pavlovian CSs predictive of monetary outcomes, and CSs representing subjects' favorite drinks. We found that these effects correlated highly both in patients and controls, suggesting that there is one central process determining the extent to which an individual subject shows PIT effects. This correlation may also partially address issues concerning the nature of the PIT effect. Both general and specific PIT processes could contribute to the effect of the CSs, given that instrumental behaviors were also rewarded with monetary outcomes. However, this is not possible for the drink CSs, and the strong correlation between the two effects suggests that the PIT effect in response to fractal CSs does at least contain a substantial general component. On an individual level, PIT effects varied very substantially between subjects, with around $60 \%$ in patients and $37 \%$ in healthy controls showing individually significant effects. This high variability between subjects might be useful if related to longitudinal outcomes of interest, but here led to rather small group effects. The present task design was based on Huys et al. [36] and Geurts et al. [39]. While the PIT effect strength and variability across subjects is comparable with the first, it is substantially stronger than the latter. Both previous studies employed healthy controls; but only the latter was performed inside an MRI scanner. It is as yet unclear precisely what factors in the tasks account for the variability across studies.

The second aim was to explore how AUD affected PIT. The current results suggest a heightened propensity to PIT, and a stronger negative PIT effect (conditioned suppression). Against our expectations, we were not able to show any difference in appetitive PIT. Theories of addiction put emphasis on striatal and prefrontal mechanisms [49], with the striatal component particularly involved in a shift towards habitual [50] or modelfree $[11,51-53]$ decision-making, and the prefrontal component resulting in a concomitant impairment of goal-directed decision-making [54; Sebold et al., this issue]. Pavlovian values are attached to stimuli, unlike in- 
strumental values, which are attached to action or stimulus-action pairs. However, pavlovian values can be both model free and model based $[14,55,56]$, producing general and specific PIT, respectively, and relying differentially on the nucleus accumbens core and shell [23]. In the setting of alcohol addiction, both model-based and model-free decision-making systems likely play important parts. On the one hand, recent work on sign tracking [57] suggests a particularly important role for model-free contributions to behavior in the nucleus accumbens core. On the basis of this, one might expect a strengthened general appetitive PIT effect to identify a certain vulnerability to drug addiction. On the other hand, work particularly involving cocaine $[54-56,58]$ might similarly argue for a stronger outcome-specific PIT component. Indeed, in the sister paper [Sebold et al., this issue], we find in another task that alcohol dependence results particularly in impairments of goal-directed decisions after nonrewards. We are not aware of work on conditioned suppression in AUD, and the results hence certainly need replication. However, we have recently found that dietary serotonin depletion selectively affects aversive PIT, and it is tempting to relate our current findings to emerging arguments about the involvement of serotonin in addiction [59].

Rather unsurprisingly, patients but not controls chose fractal CSs over alcoholic pictures in the forced choice tests. This may be influenced by social desirability (having undergone a recent detoxification), but also by currently active explicit motivation to abstain. Strikingly, however, alcohol-related CSs also appeared to suppress, rather than enhance, responding during PIT. This finding appeared to hold for both patients and controls and also attests to the consistency between preferences and PIT effects. As we pointed out, pavlovian CSs can derive value both through model-free and modelbased mechanisms. However, an outcome-specific model-based PIT account of this is difficult as instrumental and pavlovian CSs were not associated with or predictive of the same outcome. Accounting for this with a general PIT, the model-free mechanism is equally difficult and would run counter to the long-standing view that drug cues are appetitive. One answer to this might come from the temporal stability of the effect, comparing it right after detoxification and some time later. Some of the subjects failed to acquire the pavlovian contingencies. This may be due to either the delay between the training and the forced choice task (approx. 25 min apart; see also Trick et al. [34]). However, a caveat concerning the forced choice procedure is that it may tap explicit rather than implicit [60] processes, and that subjects might show evidence of PIT even in the absence of explicit knowledge [61]. However, the numbers were too small to clearly show this.

Given possible gender differences with respect to responsiveness to alcohol cues [62], we decided to focus on male participants in our pilot sample. Whereas males have a higher risk of developing alcohol dependence, lifetime prevalence numbers in females varying between 2 and $8 \%$ in Europe and the USA $[63,64]$ suggest that the identification of further gender effects with respect to contextual cues is of importance. In terms of recruiting a representative sample of alcohol-dependent patients, we will include women in future samples; however, at this point, gender effects were beyond the scope of this pilot study.

In conclusion, these pilot results suggest that our PIT paradigm is suitable for testing alcohol-dependent patients and healthy controls. We observed a stronger PIT effect in patients suffering from AUD compared to healthy controls and a high interindividual variance between subjects, which is an important factor for further studies on predicting the development and maintenance of AUD. Therefore, our PIT task might allow insights into the decision-making structure underlying the disease.

\section{Acknowledgment}

We are very grateful to Carolin Wackerhagen for help with generating the beverage stimuli, to Nadine Bernhardt for substantial help during part of the task development and implementation in the MRI scanner and to the LeAD-study teams in Dresden and Berlin. This work was supported by the German Research Foundation (Deutsche Forschungsgemeinschaft, FOR 1617: grants HE2597/14-1, SM80/7-1 and RA1047/2-1).

References

Neuropsychobiology 2014;70:111-12 119
1 Bottlender M, Spanagel R, Soyka M: One drink, one drunk - controlled drinking by alcoholics? 3-year-outcome after intensive outpatient treatment (in German). Psychother Psychosom Med Psychol 2007;57:32-38.

$\checkmark 2$ Boothby LA, Doering PL: Acamprosate for the treatment of alcohol dependence. Clin Ther 2005;27:695-714.

-3 Jones BT, McMahon J: Negative alcohol expectancy predicts post-treatment abstinence survivorship: the whether, when and why of relapse to a first drink. Addiction 1994;89: 1653-1665.

4 Jones BT, Corbin W, Fromme K: A review of expectancy theory and alcohol consumption. Addiction 2001;96:57-72.

Pavlovian-to-Instrumental Transfer in DOI: $10.1159 / 000363507$ 
5 Robins LN, Davis DH, Nurco DN: How per- $>22$ Holmes NM, Marchand AR, Coutureau E: manent was Vietnam drug addiction? Am J Public Health 1974;64(suppl 12):38-43.

-6 Everitt BJ, Robbins TW: Neural systems of reinforcement for drug addiction: from actions to habits to compulsion. Nat Neurosci $2005 ; 8$ : 1481-1489.

7 Robbins TW, Everitt BJ: Drug addiction: bad habits add up. Nature 1999;398:567-570.

8 Di Chiara G: Drug addiction as dopamine- 24 dependent associative learning disorder. Eur J Pharmacol 1999;375:13-30.

-9 Kiefer F, Dinter C: New approaches to addiction treatment based on learning and memory. 25 Curr Top Behav Neurosci 2013;13:671-684.

$\checkmark 10$ Tiffany ST, Carter BL: Is craving the source of compulsive drug use? J Psychopharmacol 1998;12:23-30.

11 Daw ND, Niv Y, Dayan P: Uncertainty-based competition between prefrontal and dorsolateral striatal systems for behavioral control. Nat Neurosci 2005;8:1704-1711.

-12 Sutton RS, Barto AG: Reinforcement Learning: An Introduction. Cambridge, MIT Press, 1998.

13 Robinson TE, Berridge KC: The incentive sensitization theory of addiction: some current issues. Philos Trans R Soc Lond Ser B Biol Sci 2008;363:3137-3146.

14 Dolan RJ, Dayan P: Goals and habits in the brain. Neuron 2013;80:312-325.

15 Schultz W, Dayan P, Montague PR: A neural substrate of prediction and reward. Science 1997;275:1593-1599.

16 Montague PR, Dayan P, Sejnowski TJ: A framework for mesencephalic dopamine systems based on predictive hebbian learning. J Neurosci 1996;16:1936-1947.

-17 Steinberg EE, Keiflin R, Boivin JR, Witten IB, Deisseroth K, Janak PH: A causal link between prediction errors, dopamine neurons and learning. Nat Neurosci 2013;16:966-973.

-18 Lesaint F, Sigaud O, Flagel SB, Robinson TE, Khamassi M: Modelling individual differences in the form of pavlovian conditioned approach responses: a dual learning systems approach with factored representations. PLoS Comput Biol 2014;10:e1003466.

19 Huys QJM, Tobler PT, Hasler G, Flagel SB: The role of learning-related dopamine signals in addiction vulnerability. Prog Brain Res 2014;211:31-77.

20 Flagel SB, Akil H, Robinson TE: Individual differences in the attribution of incentive salience to reward-related cues: implications for addiction. Neuropharmacology 2009;56 (suppl 1):139-148.

-21 Flagel SB, Robinson TE, Clark JJ, Clinton SM, Watson SJ, Seeman P, Phillips PE, Akil H: An animal model of genetic vulnerability to behavioral disinhibition and responsiveness to reward-related cues: implications for addiction. Neuropsychopharmacology 2010;35: $388-400$.
Pavlovian to instrumental transfer: a neurobehavioural perspective. Neurosci Biobehav Rev 2010;34:1277-1295.

23 Corbit LH, Balleine BW: Double dissociation of basolateral and central amygdala lesions on the general and outcome-specific forms of pavlovian-instrumental transfer. J Neurosci 2005;25:962-970.

24 Cartoni E, Puglisi-Allegra S, Baldassarre G: The three principles of action: a pavlovianinstrumental transfer hypothesis. Front Behav Neurosci 2013;7:153.

25 Cardinal RN, Parkinson JA, Hall J, Everitt BJ Emotion and motivation: the role of the amygdala, ventral striatum, and prefrontal cortex. Neurosci Biobehav Rev 2002;26:321-352.

26 Lex A, Hauber W: Dopamine D1 and D2 receptors in the nucleus accumbens core and shell mediate pavlovian-instrumental transfer. Learn Mem 2008;15:483-491.

27 Dickinson A, Smith J, Mirenowicz J: Dissociation of pavlovian and instrumental incentive learning under dopamine antagonists. Behav Neurosci 2000;114:468-483.

28 Wassum KM, Ostlund SB, Balleine BW, Maidment NT: Differential dependence of pavlovian incentive motivation and instrumental incentive learning processes on dopamine signaling. Learn Mem 2011;18:475-483.

29 LeBlanc KH, Ostlund SB, Maidment NT: Pavlovian-to-instrumental transfer in cocaine seeking rats. Behav Neurosci 2012;126:681689 .

30 LeBlanc KH, Maidment NT, Ostlund SB: Repeated cocaine exposure facilitates the expression of incentive motivation and induces habitual control in rats. PloS One 2013; 8:e61355.

31 Shiflett MW, Riccie M, Dimatteo R: The effects of amphetamine sensitization on conditioned inhibition during a pavlovian-instrumental transfer task in rats. Psychopharmacology 2013;230:137-147.

32 Corbit LH, Janak PH: Ethanol-associated cues produce general pavlovian-instrumental transfer. Alcohol Clin Exp Res 2007;31:766-774.

-33 Saddoris MP, Stamatakis A, Carelli RM: Neural correlates of pavlovian-to-instrumental transfer in the nucleus accumbens shell are selectively potentiated following cocaine selfadministration. Eur J Neurosci 2011;33: 2274-2287.

34 Trick L, Hogarth L, Duka T: Prediction and uncertainty in human pavlovian to instrumental transfer. J Exp Psychol Learn Mem Cogn 2011;37:757-765.

35 Talmi D, Seymour B, Dayan P, Dolan RJ: Human pavlovian-instrumental transfer. J Neurosci 2008;28:360-368.

36 Huys QJ, Cools R, Golzer M, Friedel E, Heinz A, Dolan RJ, Dayan P: Disentangling the roles of approach, activation and valence in instrumental and pavlovian responding. PLoS Comput Biol 2011;7:e1002028.
37 Nadler N, Delgado MR, Delamater AR: Pavlovian to instrumental transfer of control in a human learning task. Emotion 2011;11:11121123.

38 Bray S, Rangel A, Shimojo S, Balleine B, O'Doherty JP: The neural mechanisms underlying the influence of pavlovian cues on human decision making. J Neurosci 2008;28: 5861-5866.

39 Geurts DE, Huys QJ, den Ouden HE, Cools R: Aversive pavlovian control of instrumental behavior in humans. J Cogn Neurosci 2013; 25:1428-1441.

40 Prevost C, Liljeholm M, Tyszka JM, O’Doherty JP: Neural correlates of specific and general pavlovian-to-instrumental transfer within human amygdalar subregions: a high-resolution fMRI study. J Neurosci 2012;32:83838390.

-41 Sullivan JT, Sykora K, Schneiderman J, Naranjo CA, Sellers EM: Assessment of alcohol withdrawal: the revised Clinical Institute Withdrawal Assessment for Alcohol scale (CIWA-AR). Br J Addict 1989;84:1353-1357.

42 Wittchen H-U: Computer scoring of CIDI diagnoses. Int J Methods Psychiatr Res 1993;3: 101-107.

43 Brainard DH: The psychophysics toolbox. Spatial Vision 1997; 10:433-436.

44 Pelli DG: The videotoolbox software for visual psychophysics: transforming numbers into movies. Spatial Vision 1997;10:437-442.

45 Peli E: Contrast in complex images. J Opt Soc Am A Opt Image Sci 1990;7:2032-2040.

46 MATLAB version 7.12.0. Natick, MathWorks Inc, 2011.

47 IBM Corp: IBM SPSS statistics for Windows, version 20.0. Armonk, IBM Corp, 2011.

48 Robinson TE, Berridge KC: Addiction. Annu Rev Psychol 2003;54:25-53.

49 Volkow ND, Fowler JS, Wang GJ, Baler R, Telang F: Imaging dopamine's role in drug abuse and addiction. Neuropharmacology 2009;56(suppl 1):3-8.

50 Robbins TW, Gillan CM, Smith DG, de Wit S, Ersche KD: Neurocognitive endophenotypes of impulsivity and compulsivity: towards dimensional psychiatry. Trends Cogn Sci 2012; 16:81-91.

51 Redish AD, Jensen S, Johnson A: A unified framework for addiction: vulnerabilities in the decision process. Behav Brain Sci 2008;31: 415-487.

52 Hogarth L, Field M, Rose AK: Phasic transition from goal-directed to habitual control over drug-seeking produced by conflicting reinforcer expectancy. Addict Biol 2013;18:88-97.

53 Hogarth L, Balleine BW, Corbit LH, Killcross S: Associative learning mechanisms underpinning the transition from recreational drug use to addiction. Ann NY Acad Sci 2013;1282: $12-24$. 
54 Takahashi YK, Roesch MR, Wilson RC, Toreson K, O’Donnell P, Niv Y, Schoenbaum G: Expectancy-related changes in firing of dopamine neurons depend on orbitofrontal cortex. Nat Neurosci 2011;14:1590-1597.

55 Schoenbaum G, Roesch MR, Stalnaker TA Takahashi YK: A new perspective on the role of the orbitofrontal cortex in adaptive behaviour. Nat Rev Neurosci 2009;10:885-892.

56 Lucantonio F, Stalnaker TA, Shaham Y, Niv Y, Schoenbaum G: The impact of orbitofrontal dysfunction on cocaine addiction. Nat Neurosci 2012;15:358-366.

57 Flagel SB, Clark JJ, Robinson TE, Mayo L, Czuj A, Willuhn I, Akers CA, Clinton SM, Phillips PE, Akil H: A selective role for dopamine in stimulus-reward learning. Nature 2011;469:53-57.
58 McDannald MA, Lucantonio F, Burke KA, Niv Y, Schoenbaum G: Ventral striatum and orbitofrontal cortex are both required for model-based, but not model-free, reinforcement learning. J Neurosci 2011;31:2700-2705.

59 Heinz AJ, Beck A, Meyer-Lindenberg A, Sterzer P, Heinz A: Cognitive and neurobiological mechanisms of alcohol-related aggression. Nat Rev Neurosci 2011;12:400-413.

60 Knowlton BJ, Mangels JA, Squire LR: A neostriatal habit learning system in humans. Science 1996;273:1399-1402.

61 Lovibond PF, Shanks DR: The role of awareness in pavlovian conditioning: empirical evidence and theoretical implications. J Exp Psychol Anim Behav Processes 2002;28:3-26.
2 Seo D, Jia Z, Lacadie CM, Tsou KA, Bergquist $\mathrm{K}$, Sinha R: Sex differences in neural responses to stress and alcohol context cues. Hum Brain Mapp 2011;32:1998-2013.

63 Rehm J, Room R, van den Brink W, Jacobi F: Alcohol use disorders in EU countries and Norway: an overview of the epidemiology. Eur Neuropsychopharmacol 2005; 15:377388

64 Hasin DS, Stinson FS, Ogburn E, Grant BF: Prevalence, correlates, disability, and comorbidity of DSM-IV alcohol abuse and dependence in the United States: results from the national epidemiologic survey on alcohol and related conditions. Arch Gen Psychiatry 2007; 64:830-842. 